\title{
The Solution and Simulation of Air-to-air Missile Launch Envelope Based on Cooperative Air Combat of Manned/Unmanned Formation
}

\author{
Yinghao $\mathrm{Li}^{\text {a) }}$, Rong Fan \\ Aeronautics Engineering College, Air Force Engineering University, Xi'an 710038, China. \\ a)1378168095@163.com
}

\begin{abstract}
Considering the new air combat scenario involving manned/unmanned aerial vehicle cooperative combat formation, a simulation of air-to-air missile launch envelope based on cooperative air combat of manned/unmanned formation is built to resolve the new problem of cooperative air combat. Based on the mechanism of launch envelope of air-to-air missile in single-aircraft case, considering the model of cooperative launch/guidance of air-to-air missile the definition of cooperative launch envelope was conclude. The golden section-probability searching method was proposed to calculate the cooperative launch envelope and some simulations were made to work out the parameters' affection in case of cooperatively attacking. Compared simulation results indicate the unmanned/manned aerial vehicle cooperative combat formation enlarges the allowable launch envelope of missile obviously.
\end{abstract}

Keywords: unmanned aerial vehicle, air-to-air missile, allowable launch envelope, golden section-probability searching method.

\section{INTRODUCTION}

Modern air combat center is gradually changing from platform to network. With unmanned/manned aerial vehicle cooperative combat system, the launch and guidance of air-to-air missiles are no longer confined to the same platform. UAV (unmanned aerial vehicle) can greatly improve the attack condition and range of air-to-air missile by choosing reasonable strategy to fly and launch missile. The traditional attack area does not consider the flight strategy of UAV, nor does it involve cooperative factors, so it is no longer applicable to this combat system and needs to be improved.

At present, some research achievements have been made on the cooperative operations of unmanned/manned aerial vehicle, but there are few researches on the launching area of air-to-air missile under the condition of unmanned/manned aerial vehicle cooperation. In [1], the cooperative launching area of air-to-air missile under multi-fighter formation is studied, and the cooperative constraints of formation are considered, and the golden section method is used to search the missile launch area without touching the cooperation with (UAV). In [2], the paper studies the dynamic attack zone calculation and information indication of air-to-air missile after ejection, which is similar to unmanned aerial vehicle (UAV) preflight attack. In [3], the literature studies aim at the allowable launch envelope of an air-to-air missile.

In this paper, the cooperative attack mode with unmanned/manned aerial vehicle is analyzed, and the cooperative launch area with unmanned/manned aerial vehicle is defined. The simulation model of three degrees of freedom is used to calculate the fighter, target and missile. Aiming at the dead cycle that the golden section method may fall into in the course of searching the boundary, the improvement measures are put forward. On this basis, the distant and near boundary of the launching area is searched, and the influence of the target parameters on the boundary of the launching area is explored. 


\title{
THE CONCEPT OF AIR-TO-AIR MISSILE ALLOWABLE LAUNCH ENVELOPE ABOUT UNMANNED/MANNED AERIAL VEHICLE COOPERATIVE COMBAT FORMATION
}

\author{
Single Platform Air-to-Air Missile Allowable Launch Envelope
}

A single platform air-to-air missile is allowed to use the attack aircraft as a reference to meet certain attack conditions, and the range of the initial position of the target can be hit by the attack plane with a certain probability. [4-5] The launching area is mainly determined by the parameters of the launching platform and missile. The launching area is centered on the attack aircraft and represents the three-dimensional space area which can reach the target's initial position with a certain probability.

\section{Unmanned/Manned Aerial Vehicle Attack Mode}

There is a unmanned/manned aerial vehicle cooperative attack mode, that is, the UAV, by virtue of its small size and fast speed, can protrude rapidly in the event that it is about to catch up with the enemy, and then under cooperative conditions. A new attack mode is developed from the traditional single-plane launch / guidance system under the condition of data link support, which includes fighter detection and tracking, UAV launch and the fighter guided. The main characteristics of UAV are as follows: there are the fighter detection and tracking target, UAV receives target data by inter-aircraft data link, and launches air-to-air missile against target.

\section{The Launching Mode}

Therefore, the air-to-air missile launch mode with manned / unmanned aerial vehicle (UAV) is different from the traditional platform aiming at the launch condition of missile and the launch / guidance method of the missile.

\section{Missile Formation and Launch Condition}

Whether the missile can be launched can be determined by "whether the aircraft is stable to track and intercept the target" and "whether the target enters the missile's permitted firing zone". [8] For the aircraft carrying out the attack, the former is closely related to the performance of airborne radar, and the latter is related to the attack distance and maneuverability of the air-to-air missile.

With the cooperation of manned / unmanned aerial vehicle, the radar is used to detect, track and intercept the target. The UAV launches missiles according to the information of the fighter and realizes the separation of detection and tracking and weapon launching platform.

\section{Missile Launch / Guidance Mode}

Under the cooperative attack mode with manned / unmanned aerial vehicle, the traditional attack mode of single launch / guidance has developed into the attack mode of UAV launch / fighter guidance. In this mode, the target is detected, tracked and intercepted by the fighter, and the target information is shared by the data link between the aircraft, and the air-to-air missile is launched by the UAV. The target is continuously irradiated by the fighter and guided in the middle.

\section{Air-to-Air Missile Launch Envelope}

In combination with the above conditions, a manned / unmanned aerial vehicle cooperative air-to-air missile launching area can be defined as a manned / unmanned aerial vehicle synergistic condition. When the attack conditions are met, the attack machine or the UAV launch air-to-air missile can hit the initial position of the target. The launch area is a superposition of manned / unmanned aerial vehicle allowable launch envelope 


\section{MOTION MODEL}

\section{Fighter Motion Model}

Taking fighter aircraft as an example, the equations of motion parameters are established.

$$
\left\{\begin{array}{l}
\dot{x}_{z}=v_{z} \cos \theta_{z} \sin \psi_{z} \\
\dot{y}_{z}=v_{z} \sin \theta_{z} \\
\dot{z}_{z}=-v_{z} \cos \theta_{z} \cos \psi_{z} \\
\dot{v}_{z}=n_{z x} g \\
\dot{\theta}_{z}=n_{z y} g / v_{z} \\
\dot{\psi}_{z}=n_{z z} g /\left(v_{z} \cos \theta_{z}\right)
\end{array}\right.
$$

Where $x_{z}, y_{z}, z_{z}$ refer to the position of fighter in inertial coordinate system. $v_{z}, \theta_{z}, \psi_{z}$ refer to the velocity, bank angle and heading angle; $n_{z x} 、 n_{z y} 、 n_{z z}$ refer to longitudinal control overload for fighter aircraft, yaw and pitch turn control overload.

In the same way, the target motion parameter equation and UAV motion parameter equation can be established by referring to the motion parameter equation of fighter plane.

\section{Air-to-Air Missile Motion Model}

$$
\left\{\begin{array}{l}
\dot{x}_{m}=v_{m} \cos \theta_{m} \sin \psi_{m} \\
\dot{y}_{m}=v_{m} \sin \theta_{m} \\
\dot{z}_{m}=v_{m} \cos \theta_{m} \cos \psi_{m} \\
\dot{v}_{m}=(F-P) / m-g \sin \theta_{m} \\
\dot{\theta}_{m}=\left(n_{m y}-\cos \theta_{m}\right) g / v_{m} \\
\dot{\psi}_{m}=-n_{m z} g /\left(v_{m} \cos \theta_{m}\right)
\end{array}\right.
$$

Where $x_{m}, y_{m}, z_{m}$ refer to position of missile in inertial coordinate system $v_{m} 、 \theta_{m} 、 \psi_{m}$ refer to the velocity, bank angle and heading angle; $n_{m y} 、 n_{m z}$ refer to control overload for missile yaw and pitch turn respectively, $F 、 P 、 m$ refer to missile thrust, air resistance and mass.

The law of their change is:

$$
\begin{aligned}
& F= \begin{cases}\bar{F} & t \leq t_{0} \\
0 & t>t_{0}\end{cases} \\
& P=\left\{\frac{1}{2} \rho v_{m}^{2} S_{m} C_{m}\right. \\
& m=\left\{\begin{array}{lc}
m_{0}-m_{\mathrm{sec}} t & t \leq t_{0} \\
m_{0}-m_{\mathrm{sec}} t_{0} & t>t_{0}
\end{array}\right.
\end{aligned}
$$

Where $\rho$ refer to atmosphere density. $S_{m}$ is effective area of projectile, $C_{m}$ is missile resistance coefficient, $m_{\text {sec }}$ is fuel second flow, $m_{0}$ is missile initial launching mass, $t_{0}$ is maximum operating time of missile engine. 


\section{Relative Motion Model}

In the study of missile guidance process, targeting, tracking and launching of UAV missiles, the relative equations of motion of target-fighter, target-UAV, and missile-fighter line of sight are usually used.

The relative motion equation is established by taking the target - fighter as an example.

$$
\left\{\begin{array}{l}
r_{z t x}=x_{t}-x_{z} \\
r_{z t y}=y_{t}-y_{z} \\
r_{z t z}=z_{t}-z_{z} \\
r_{z t}=\sqrt{r_{z t x}^{2}+r_{z t y}^{2}+r_{z t z}^{2}} \\
\psi_{z t}=\arctan \left(-r_{z t z} / r_{z t x}\right) \\
\theta_{z t}=\arcsin \left(r_{z t y} / r_{z t}\right)
\end{array}\right.
$$

The position vector $\mathrm{r}$, including $\left(r_{z t}, \psi_{z t}, \theta_{z t}\right)$, of target fighter line of sight coordinate system is set up, and the relative motion equation of target UAV and missile fighter is established according to this method. Derivative it to time D

$$
\left\{\begin{array}{l}
\dot{r}_{z t x}=x_{t}-x_{z} \\
\dot{r}_{z t y}=y_{t}-y_{z} \\
\dot{r}_{z t z}=z_{t}-z_{z} \\
\dot{r}_{z t}=\left(r_{z t x} \dot{r}_{z t x}+r_{z t y} \dot{r}_{z t y}+r_{z t z} \dot{r}_{z t z}\right) / r_{z t} \\
\dot{\psi}_{z t}=\left(r_{z t z} \dot{r}_{z t x}-r_{z t z} \dot{r}_{z t x}\right) /\left(r_{z t x} \dot{r}_{z t x}+r_{z t z} \dot{r}_{z t z}\right) \\
\dot{\theta}_{z t}=\left(r_{z t} \dot{r}_{z t y}-r_{z t y} r_{z t}\right) /\left(r_{z t} \times \sqrt{r_{z t} r_{z t}-r_{z t y} r_{z t y}}\right)
\end{array}\right.
$$

Where the derivative $\dot{r}_{z t} 、 \dot{\psi}_{z t} 、 \dot{\theta}_{z t}$ representing the position vector $\mathrm{r}$.

\section{Missile Guidance Model}

In this model, the proportional guidance method is used to guide the missile. Under the control of the proportional guidance method, the direction change rate of the missile velocity is proportional to the missile line of sight rate. [6]

$$
\left\{\begin{array}{l}
K \dot{\psi}_{m t}=\dot{\psi}_{m} \\
K \dot{\theta}_{m t}=\dot{\theta}_{m}
\end{array}\right.
$$

The guidance coefficient K needs to satisfy the convergence condition of an airborne missile.

$$
K>\frac{2|\dot{r}|}{v_{m} \cos \mu}
$$

Where $\mu$ is the forward vector angle of the airborne missile.

Missile seeker usually has maximum tracking angular velocity $\omega_{\max }$ limit 


$$
\dot{q}=\left\{\begin{array}{cc}
\omega_{\max } \operatorname{sgn}(\dot{q}) & |\dot{q}| \geq \omega_{\text {max }} \\
\dot{q} & |\dot{q}|<\omega_{\text {max }}
\end{array}\right.
$$

Missile control overload $\bar{n}_{1}=v_{m} \dot{q}_{m t} K / g$ needs to meet the maximum overload limit,

$$
\bar{n}= \begin{cases}\bar{n}_{1} & \left|\bar{n}_{1}\right| \leq n_{k} \\ n_{k} \operatorname{sgn}\left(\bar{n}_{1}\right) & \left|\bar{n}_{1}\right|>n_{k}\end{cases}
$$

Consider the delay of the rudder [7],

$$
\dot{n}=\left(\bar{n}-n_{i-1}\right) / \tau
$$

Where $n_{i-1}$ is the overload in the last time. $\tau$ is the reaction time ranging from $\tau=20 \sim 30 \mathrm{~ms}$, now the overload $n_{i}$

$$
n_{i}=n_{i-1}+\dot{n} \times \Delta t
$$

Where $\Delta t$ is the control step [1] .

\section{Cooperative Emission Region Constraint}

During missile flight, at time $t$, if $r<e$ and $t>t_{j}$, We think the missile hit the target or the attack failed, $e$ is Allowable miss distance, $t_{j}$ is missile defuse time.

At the same time, the missile must satisfy the flight height restriction, the minimum controllable flight speed limit, the controllable flight time limit and the maximum field angle constraint,

$$
\left\{\begin{array}{c}
y_{m} \geq 200 \mathrm{~m} \\
v_{m} \geq 50 \mathrm{~m} / \mathrm{s} \\
t \leq t_{j} \\
\theta_{m t} \leq \theta_{m t \max }
\end{array}\right.
$$

Where $\theta_{m t \max }$ is missile maximum field of view angle.

\section{Fighter and Unmanned Aerial Vehicle Formation Constraints}

At the same time, the Fighter and UAV formation must have detection range constraint, detection angle constraint, maximum range constraint, mid-guidance distance and angle constraint. 


$$
\left\{\begin{array}{l}
r_{z t} \leq R_{t c} \\
r_{z w} \leq R_{x t} \\
r_{w t} \leq R_{z d} \\
\delta_{z t} \leq \delta_{z d} \\
\theta_{z t} \leq \theta_{z t \max }
\end{array}\right.
$$

Where $R_{t c}$ refer to maximum detectable range, $R_{x t}$ is maximum cooperative flight distance, $R_{z d}$ is maximum mid-guidance distance, $\delta_{z d}$ is maximum guidance angle, $\theta_{z t \max }$ is maximum detection angle.

\section{SIMULATION OF ALLOWABLE LAUNCH ENVELOPE}

To solve the cooperative launch area of fighter UAV, the surrounding space is searched first to determine whether the target is in an initial position. If the UAV launches an air-to-air missile, it can hit the target. The simulation first establishes the polar coordinate system centered on the fighter plane, then gives the coordinates of the UAV, the coordinates of the UAV are calculated according to the location of the target, and then the onedimensional golden section method [9-10] is used to solve the surrounding area of the fighter aircraft. Search for missile attack near and long-range boundaries.

\section{Golden Section-Probability Searching Method}

Select a certain direction in the angle of view of the fighter, select the range of initial distance $\left[a_{0}, b_{0}\right]$, calculate the golden section point $R_{g}=a_{0}+0.618 \times\left(b_{0}-a_{0}\right)$, then carry on the simulation analysis to a point $R_{g}$, study whether the missile can hit the target of this point under the constraint condition. If missile hit it, then $a_{i}=R_{g}, b_{i}=b_{i-1}$ otherwise $a_{i}=a_{i-1}, b_{i}=R_{g}$, This method is applied to the circular calculation until the distant boundary value $b_{i}$ satisfying the accuracy requirement is obtained. When missile hit it, then $a_{i}=a_{i-1}, b_{i}=R_{g}$, otherwise $a_{i}=R_{g}, b_{i}=b_{i-1}$, According to this method, the near boundary value $a_{i}$ satisfying the precision requirement can be obtained. And then gradually change the angle and search the entire field of view.

\section{Defects and Improvements}

If the golden section method is to iterate normally, the initial point must be larger than the near boundary when calculating the far boundary, otherwise, the value of its far boundary will approach to 0 , and the initial point must be smaller than the far boundary when calculating the near boundary, otherwise, the near boundary will approach infinity. This is because the non-emissivity of the carrier is divided into the short-range non-emissive region and the long-range non-emissive zone, usually from near too far in order of the close-range non-emissive region, the launching area, the long-range non-emissive area. The golden section method can only search between one nonemissive region and the emissive zone. When the initial point falls into another non-emissive zone, it will fall into a dead cycle.

Algorithm 1 Near boundary golden section-probability searching method

Initialize $[\mathrm{a} 0, \mathrm{~b} 0]$

Initialize Shit Severhit,i

while $\mid$ bi-ai $\mid>\varepsilon$ do

$\mathrm{Rg}=\mathrm{ai}+($ bi-ai) $* 0.618$

Severhit $=f(\operatorname{Rg}) \|$ Shit 
if Severhit=false then

ai=Rg, bi=bi-1

Shit $=\mathrm{f}(\mathrm{Rg})$

else if Shit=false then

bi=Rg, ai=ai-1

else ai $=$ Rg, bi $=$ bi-1

end if

$\mathrm{i}=\mathrm{i}+1$

output:(bi)

Where Shit refer to the missile whether can hit the target or not, Severhit refer to the missile whether hit the target or not in the past time, $\mathrm{f}(\mathrm{x})$ refer to the missile whether hit the target or not on the condition of $\mathrm{x}$. As shown in Algorithm 1, the improved approach is described by taking the near boundary as an example.

\section{Simulation Flow}

According to the simulation flow chart shown in following, the missile launching area under the cooperation of unmanned/manned aerial vehicle is simulated and calculated.

Step 1: Initialize the parameters, the velocity, bank angle and heading angle $v_{m}, \theta_{m}, \psi_{m}$.

Step 2: Based on the detection range and missile attack distance, initialize [a0, b0].

Step 3: Calculate the golden section point $R_{g}=a_{0}+0.618 \times\left(b_{0}-a_{0}\right)$.

Step 4: Based on the position of target, fighter and UAV, we simulate the missile attack the target. The target, fighter and UAV motive as the motion model. The radar detection constraint and guidance rate calculation and constraint would be calculated.

Step 5: If unmanned/manned aerial vehicle can't satisfy the constraint, bi=Rg, ai=ai-1, else we need judge the missile attack result. If the missile hit the target, then ai=Rg, bi=bi-1, otherwise $\mathrm{bi}=\mathrm{Rg}, \mathrm{ai}=\mathrm{ai}-1$.

Step 6: If the error between the near boundary and far boundary is in the precision range, we need store the boundary value b, else we return to Step 3 .

Step 7: Increasing the bank angle and heading angle according to step size and return to Step 2 until traverse all angles.

\section{ALLOWABLE LAUNCH ENVELOPE SIMULATION}

TABLE 1. The initial value of simulation

Target speed

Airborne missile engine working hours

Missile maximum power supply time

Missile fuse unlock time

Missile killing radius

Maximum field of view angle of seeker

Missile initial mass

Fuel second flow

Average thrust

Maximum motor overload

Flight speed of unmanned aerial vehicle

Flight speed

$100 \mathrm{~m} / \mathrm{s}$
$8.4 \mathrm{~S}$
$26 \mathrm{~s}$
$2 \mathrm{~s}$
$5 \mathrm{~m}$
$60^{\circ}$
$300 \mathrm{~kg}$
$10 \mathrm{~kg} / \mathrm{s}$
$6200 \mathrm{~N}$
$18 \mathrm{~g}$
$500 \mathrm{~m} / \mathrm{s}$
$200 \mathrm{~m} / \mathrm{s}$

The initial value of simulation in Tab. 1

Simulation 1: The UAV is flying directly north 

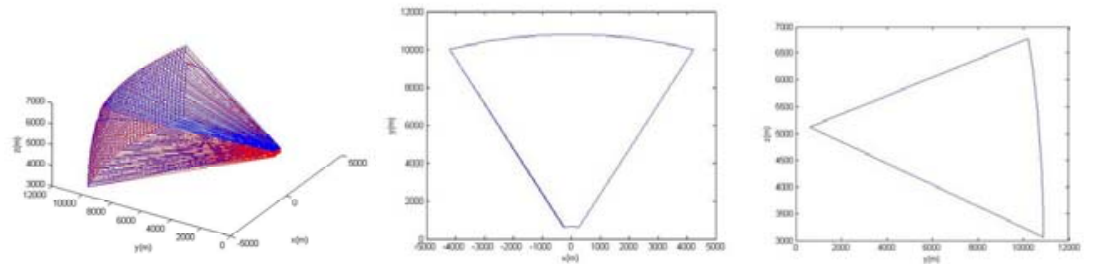

3D emitter simulation

Emission region $\mathrm{x}-\mathrm{y}$ plane diagram Emission zone $\mathrm{y}-\mathrm{z}$ plane diagram

FIGURE 1. UAV $t=0 \mathrm{~s}$, entrance angle $q=0^{\circ}$, altitude $h=5000 \mathrm{~m}$
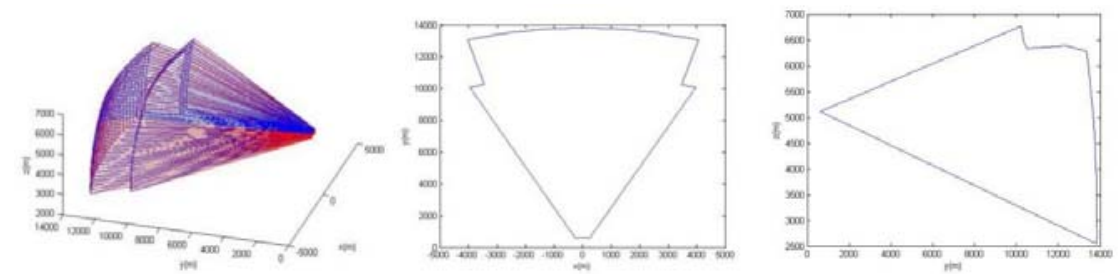

3D emitter simulation Emission region $\mathrm{x}-\mathrm{y}$ plane diagram Emission zone $\mathrm{y}-\mathrm{z}$ plane diagram

FIGURE 2. UAV $t=30 \mathrm{~s}$, entrance angle $q=0^{\circ}$, altitude $h=5000 \mathrm{~m}$

Fig. 1 compared to Fig. 2, In the horizontal plane, the far boundary of unmanned/manned aerial vehicle cooperative launch area increases, and in the vertical plane, the lower the attack boundary is, the lower the target height is, the greater the energy the missile can obtain, and the farther the attack distance is. However, the upper boundary and height change are nonlinear due to the angle limitation of the loading mechanism and the overload limitation of the missile.

Simulation 2: Entrance angle of target $q=20^{\circ}$
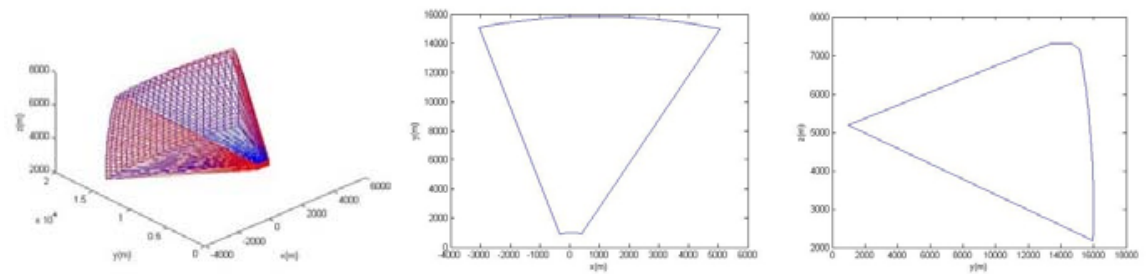

3D emitter simulation Emission region $x-y$ plane diagram Emission zone y-z plane diagram

FIGURE 3. UAV $t=0 \mathrm{~s}$, entrance angle $q=20^{\circ}$, altitude $h=5000 \mathrm{~m}$
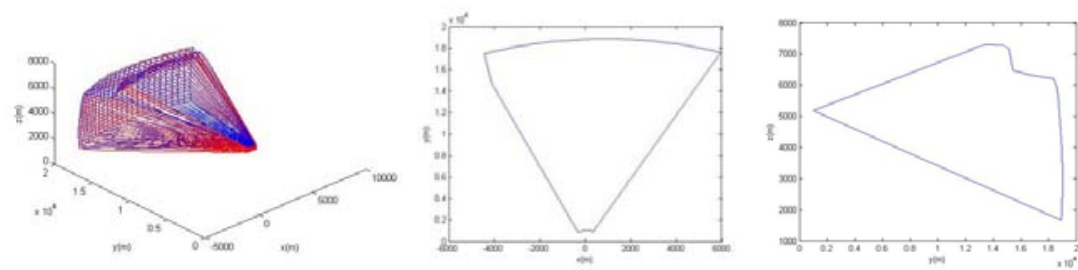

3D emitter simulation Emission region $\mathrm{x}-\mathrm{y}$ plane diagram Emission zone $\mathrm{y}-\mathrm{z}$ plane diagram

FIGURE 4. UAV $t=30 \mathrm{~s}$, entrance angle $q=20^{\circ}$, altitude $h=5000 \mathrm{~m}$

Fig. 3 is compared with Fig. 4, and the result is similar to that above. However, because of the entry angle of the target, the gain of the UAV pre-launched missile on the left and right sides of the launch zone is not the same. 

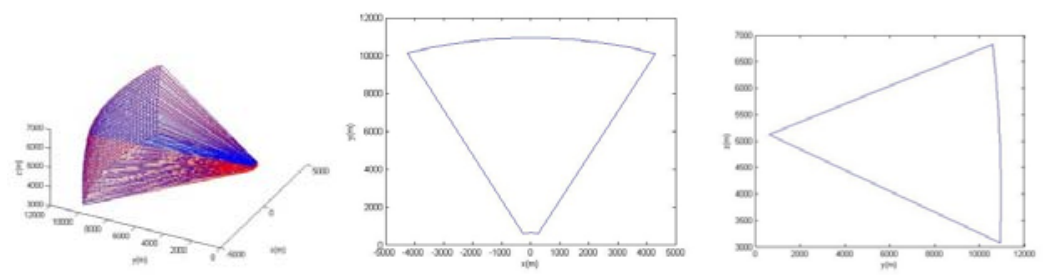

3D emitter simulation Emission region $\mathrm{x}-\mathrm{y}$ plane diagram Emission zone y-z plane diagram

FIGURE 5. UAV $t=0 \mathrm{~s}$, entrance angle $q=0^{\circ}$, altitude $h=6000 \mathrm{~m}$
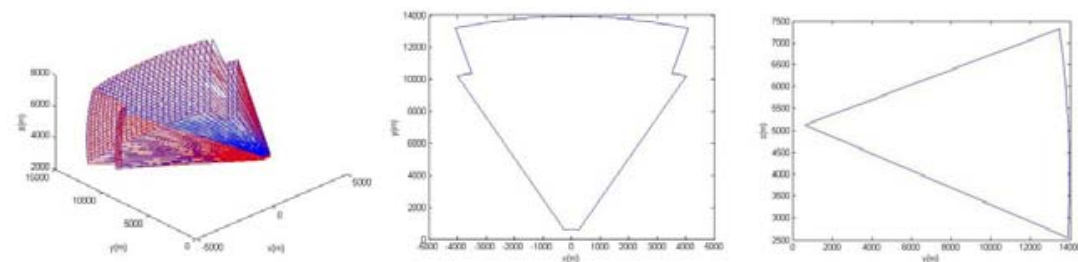

3D emitter simulation Emission region $\mathrm{x}-\mathrm{y}$ plane diagram Emission zone $\mathrm{y}-\mathrm{z}$ plane diagram

FIGURE 6. UAV $t=30 \mathrm{~s}$, entrance angle $q=0^{\circ}$, altitude $h=6000 \mathrm{~m}$

If other conditions are the same and the altitude increases, the effect on the far boundary of UAV is small, but the gain of missile upper boundary is larger.

\section{SUMMARY}

In this paper, the air-to-air missile launching area under the cooperative situation of unmanned/manned aerial vehicle is defined, the calculation model of cooperative attack area is improved, and the golden section method is improved. The simulation results show that the cooperative attack area is obviously larger than the traditional attack area, which provides the basis for the cooperative combat decision of unmanned/manned aerial vehicle.

\section{REFERENCE}

1. Diao Xinghua, Fang Yangwang, Wu Youli, Fu Xiaofei. Simulation analysis on air-to-air missile allowable launch envelope about cooperative air combat of multi-fighter formation. Journal of Beijing University of Aeronautics and Astronautics, 2014, 40(3): 370-376. (In Chinese).

2. WU Shengliang, NAN Ying. The Calculation of Dynamical Attack Zone of Air-to-air Missile after Being Launched [J]. 2013, 5:49-54. (In Chinese).

3. Kou Yingxin, Fu Zhaowang, Feng Guoqiang, et al. Simulation analysis on air-to-air missile allowable launch envelope in networked targeting[J]. Journal of Air Force Engineering University: Natural Science Edition,2012,13(2):24-28(in Chinese).

4. Chen Baolin. The theory and algorithms of optimization [M]. Beijing: Tsinghua University Press, 2007 (in Chinese).

5. Karelahti J, Virtanen K, Raivio T. Near-optimal missile avoidance trajectories via receding horizon control Journal of Guidance, Control and Dynamics,2007,30(5): 1287 -1298.

6. Siouris G M. Missile guidance and control systems [M] New York: Springer-Verlag, 2004.

7. Zhang Ping,Fang Yangwang, Qiao Zhijun, et al. A method for calculating allowable launch envelope of overthe-horizon air-to-air missile[J].Electronics Optics \& Control,2012,19(3):12-16(in Chinese).

8. Dhar A,Ghose D. Capture region for a realistic TPN guidance law [J].IEEE Transactions on Aerospace and Electronic Systems, 1993,29( 3) : 995-1003.

9. Ghose D. Capture region for true proportional navigation guidance with nonzero miss-distance [J]. Journal of Guidance, Control and Dynamics, 1994,17(3): 627-628. 
10. Zhang Ping, Fang Yangwang, Jin Chong,et al.A new method of real-time calculation about air-to-air missile launch envelopes[J].Journal of Ballistics,2010,22(4):11-14(in Chinese). 